\title{
Analysis of potential evapotranspiration using limited weather data
}

\author{
Mohammad Valipour
}

Received: 10 May 2014/ Accepted: 9 September 2014/Published online: 27 September 2014

(c) The Author(s) 2014. This article is published with open access at Springerlink.com

\begin{abstract}
The most important weather variations are temperature $(T)$, relative humidity $(\mathrm{RH})$, and wind speed (u) for evapotranspiration models in limited data conditions. This study aims to compare three $T$-based formula, $T /$ RH-based formula, and $T / \mathrm{RH} / u$-based formula to detect the performance of them under limited data and different weather conditions. For this purpose, weather data were gathered from 181 synoptic stations in 31 provinces of Iran. The potential evapotranspiration was compared with the FAO Penman-Monteith method. The results showed that $T$-based formula, $T / \mathrm{RH}$-based formula, and $T / \mathrm{RH} / u$-based formula estimated potential evapotranspiration with $R^{2}$ $>0.93$ for 6,12 , and 30 provinces of Iran, respectively. They are more suitable for southeast of Iran (YA, KE, SB, and SK). The best precise method was the $T / \mathrm{RH} / u$-based formula for SK and GO. Finally, a list of the best performance of each method has been presented to use other regions and next researches according to values of temperature, relative humidity, and wind speed. The best weather conditions to use the formulas are $14-26^{\circ} \mathrm{C}$ and $2.50-3.50 \mathrm{~m} / \mathrm{s}$ for temperature and wind speed, respectively.
\end{abstract}

Keywords Evapotranspiration · Humidity · Iran · Radiation · Temperature $\cdot$ Wind

M. Valipour $(\bowtie)$

Department of Water Engineering, Kermanshah Branch, Islamic Azad University, Kermanshah, Iran

e-mail: vali-pour@hotmail.com

\section{Introduction}

The most important weather parameters are temperature, relative humidity, and wind speed for evapotranspiration models. A review is needed to find weak points of the previous studies.

Shi et al. (2008) compared the Priestley-Taylor method with Katerji-Perrier and Todorovic methods in northeastern China. The Priestley-Taylor method, being site dependent and the simplest approach, was effective enough to estimate large time-scale (at least daily) evapotranspiration. Xu and Singh (2000) compared the Abtew, Hargreaves, Makkink, Priestley-Taylor, and Turc to estimate evaporation at Changins station in Switzerland. The Makkink and modified Priestley-Taylor equations resulted in monthly evaporation values that agreed most closely with pan evaporation in the study region. Al-Ghobari (2000) compared the Jensen-Haise and Blaney-Criddle methods to estimate potential evapotranspiration for southern region of Saudi Arabia. The results indicated that no one method provided the best results under all weather conditions. Jacobs et al. (2010) estimated the potential evapotranspiration by the Makkink method successfully. They used 80 years of meteorological observations at Wageningen, the Netherlands. However, the Priestley-Taylor model was only able to yield a fair estimation of the reference evapotranspiration during some periods of the growing season even though the soil moisture effect is integrated into the Priestley-Taylor parameter ( $\mathrm{Li}$ et al. 2011). Meanwhile, care should be taken when applying the Priestley-Taylor equation in the semiarid climate in north China. Temporally, it can be used in July and August and at daily time scale in these 2 months, but unsatisfactorily in other months and at yearly time scale (Xiaoying and Erda 2005). Furthermore, Ye et al. (2009) showed that the 
Table 1 Position of all provinces and synoptic stations

\begin{tabular}{|c|c|c|c|c|}
\hline Province & $\begin{array}{l}\text { Latitude } \\
(\mathrm{N})\end{array}$ & $\begin{array}{l}\text { Longitude } \\
\text { (E) }\end{array}$ & $\begin{array}{l}\text { Data measured } \\
\text { (year) }\end{array}$ & $\begin{array}{l}\text { Number of } \\
\text { stations }\end{array}$ \\
\hline $\mathrm{AL}$ & $35^{\circ} 55^{\prime}$ & $50^{\circ} 54^{\prime}$ & 20 & 1 \\
\hline AR & $38^{\circ} 15^{\prime}$ & $48^{\circ} 17^{\prime}$ & 30 & 4 \\
\hline BU & $28^{\circ} 59^{\prime}$ & $50^{\circ} 50^{\prime}$ & 55 & 5 \\
\hline CB & $32^{\circ} 17^{\prime}$ & $50^{\circ} 51^{\prime}$ & 51 & 4 \\
\hline EA & $38^{\circ} 05^{\prime}$ & $46^{\circ} 17^{\prime}$ & 55 & 10 \\
\hline ES & $32^{\circ} 37^{\prime}$ & $51^{\circ} 40^{\prime}$ & 55 & 12 \\
\hline FA & $29^{\circ} 32^{\prime}$ & $52^{\circ} 36^{\prime}$ & 55 & 9 \\
\hline GH & $36^{\circ} 15^{\prime}$ & $50^{\circ} 03^{\prime}$ & 47 & 2 \\
\hline GI & $37^{\circ} 15^{\prime}$ & $49^{\circ} 36^{\prime}$ & 50 & 4 \\
\hline GO & $36^{\circ} 51^{\prime}$ & $54^{\circ} 16^{\prime}$ & 54 & 3 \\
\hline HA & $34^{\circ} 52^{\prime}$ & $48^{\circ} 32^{\prime}$ & 55 & 4 \\
\hline $\mathrm{HO}$ & $27^{\circ} 13^{\prime}$ & $56^{\circ} 22^{\prime}$ & 49 & 9 \\
\hline IL & $33^{\circ} 38^{\prime}$ & $46^{\circ} 26^{\prime}$ & 20 & 3 \\
\hline $\mathrm{KB}$ & $30^{\circ} 50^{\prime}$ & $51^{\circ} 41^{\prime}$ & 19 & 1 \\
\hline $\mathrm{KE}$ & $30^{\circ} 15^{\prime}$ & $56^{\circ} 58^{\prime}$ & 55 & 8 \\
\hline $\mathrm{KH}$ & $31^{\circ} 20^{\prime}$ & $48^{\circ} 40^{\prime}$ & 55 & 14 \\
\hline KO & $35^{\circ} 20^{\prime}$ & $47^{\circ} 00^{\prime}$ & 47 & 7 \\
\hline $\mathrm{KS}$ & $34^{\circ} 21^{\prime}$ & $47^{\circ} 09^{\prime}$ & 55 & 6 \\
\hline LO & $33^{\circ} 26^{\prime}$ & $48^{\circ} 17^{\prime}$ & 55 & 9 \\
\hline MA & $34^{\circ} 06^{\prime}$ & $49^{\circ} 46^{\prime}$ & 51 & 4 \\
\hline $\mathrm{MZ}$ & $36^{\circ} 33^{\prime}$ & $53^{\circ} 00^{\prime}$ & 55 & 7 \\
\hline NK & $37^{\circ} 28^{\prime}$ & $57^{\circ} 16^{\prime}$ & 24 & 1 \\
\hline QO & $34^{\circ} 42^{\prime}$ & $50^{\circ} 51^{\prime}$ & 20 & 1 \\
\hline RK & $36^{\circ} 16^{\prime}$ & $59^{\circ} 38^{\prime}$ & 55 & 12 \\
\hline SB & $29^{\circ} 28^{\prime}$ & $60^{\circ} 05^{\prime}$ & 55 & 8 \\
\hline SE & $35^{\circ} 35^{\prime}$ & $53^{\circ} 33^{\prime}$ & 55 & 4 \\
\hline SK & $32^{\circ} 52^{\prime}$ & $59^{\circ} 12^{\prime}$ & 51 & 3 \\
\hline $\mathrm{TE}$ & $35^{\circ} 41^{\prime}$ & $51^{\circ} 19^{\prime}$ & 55 & 8 \\
\hline WA & $37^{\circ} 32^{\prime}$ & $45^{\circ} 05^{\prime}$ & 55 & 8 \\
\hline YA & $31^{\circ} 54^{\prime}$ & $54^{\circ} 17^{\prime}$ & 54 & 6 \\
\hline ZA & $36^{\circ} 41^{\prime}$ & $48^{\circ} 29^{\prime}$ & 51 & 4 \\
\hline
\end{tabular}

Table 2 Method used (FPM) and parameters applied

\begin{tabular}{lclc}
\hline Model & Reference(s) & Formula & Parameters \\
\hline FAO & Allen et al. & ET $_{\mathrm{o}}=\frac{0.408\left(R_{\mathrm{n}}-G\right)+\gamma \frac{900}{T+27} u\left(e_{\mathrm{s}}-e_{\mathrm{a}}\right)}{\Delta+\gamma(1+0.34 u)}$ & $H, \varphi, T$, \\
Penman- & $(1998)$ & & $T_{\min }$, \\
Monteith & & & $T_{\max }, \mathrm{RH}$, \\
& & & $u, n$ \\
\hline
\end{tabular}

$\mathrm{ET}_{\mathrm{o}}$ is the reference crop evapotranspiration $\left(\mathrm{mm} /\right.$ day), $R_{\mathrm{n}}$ is the net radiation $\left(\mathrm{MJ} / \mathrm{m}^{2} /\right.$ day $), G$ is the soil heat flux $\left(\mathrm{MJ} / \mathrm{m}^{2} /\right.$ day $), \gamma$ is the psychrometric constant $\left(\mathrm{kPa} /{ }^{\circ} \mathrm{C}\right), e_{\mathrm{s}}$ is the saturation vapor pressure $(\mathrm{kPa}), e_{\mathrm{a}}$ is the actual vapor pressure $(\mathrm{kPa}), \Delta$ is the slope of the saturation vapor pressure-temperature curve $\left(\mathrm{kPa} /{ }^{\circ} \mathrm{C}\right), T$ is the average daily air temperature $\left({ }^{\circ} \mathrm{C}\right), u$ is the mean daily wind speed at $2 \mathrm{~m}$ $(\mathrm{m} / \mathrm{s}), H$ is the elevation $(\mathrm{m}), \varphi$ is the latitude $(\mathrm{rad}), T_{\min }$ is the minimum air temperature $\left({ }^{\circ} \mathrm{C}\right), T_{\max }$ is the maximum air temperature $\left({ }^{\circ} \mathrm{C}\right), \mathrm{RH}$ is the average relative humidity $(\%)$, and $n$ is the actual duration of sunshine $(\mathrm{h})$
Priestley-Taylor method was more suitable for Tibetan Plateau in the absence of the parameters necessary for the calculation of the FPM. In the other research, Rojas and Sheffield (2013) showed that the radiation-based equations tended to underestimate by as much as $10 \%$, whereas the temperature-based Hargreaves model overestimated by $8 \%$ during the growing season.

Furthermore, the researchers have studied Iran to detect the best models for estimating the potential evapotranspiration (Rahimi et al. 2014; Valipour 2014a, b, c, d, e, f, g, h, i, j, k, 1, m, n; Valipour and Eslamian 2014). However, in all of the previous studies, one or more of the radiation/mass transfer/temperature-based methods have been compared with other-based methods and in the most of the cases, only one of radiation, mass transfer, or temperature-based models estimated the potential evapotranspiration better than the other methods. Moreover, the results of previous studies are not useable for estimation of the potential evapotranspiration in other regions. Because they were recommended for one or more climatic conditions, but a climatic condition contains a wide range of magnitude of each weather parameter and results of each research are not applicable for other regions without determining specified ranges of each weather parameter even if climatic conditions are identical for both regions. In addition, the governments cannot schedule for irrigation and agricultural water management when the potential evapotranspiration is estimated for a basin, wetland, watershed, or catchment instead a state or province and/or number of weather stations used is low. Since, this study aims to estimate the potential evapotranspiration for 31 provinces of Iran using average data of 181 synoptic stations and by three $T$-based formula, $T / \mathrm{RH}$-based formula, and $T / \mathrm{RH} / u$-based formula to estimate the potential evapotranspiration in limited data conditions based on the weather conditions of each province. The results help to predict evapotranspiration while there is only one or there are two/three weather variations for the study area on the basis of recorded data.

\section{Materials and methods}

In this study, weather information (from 1951 to 2010) has been gathered from 181 synoptic stations of 31 provinces in Iran. Table 1 shows position of each province and number of stations.

In each station, average of weather data in years measured has been considered as value of that weather parameter in each month (e.g. value of solar radiation in June for NK is average of 24 data gathered). A spatial interpolation method is usually used to obtain an averaged value from stations. However, most of the synoptic stations 
have been distributed in north, south, west, and east of each province based on different weather conditions and considering equal spatial distances to skip spatial interpolation method. Therefore, average of data in all stations has been considered as value of that weather parameter in each month for provinces with more than one station (e.g. value of relative humidity in June for $\mathrm{KH}$ is average of $55 \times 14=770$ data gathered). All of the data mentioned have been used to estimate the potential evapotranspiration using three $T$-based formula, $T / \mathrm{RH}$-based formula, and $T /$ $\mathrm{RH} / u$-based formula and were compared with FAO Penman-Monteith (FPM) method to determine the best method based on the weather conditions of each province (Table 2).

The best method for each province and the best performance of each method were determined using the below error indices:

$$
\begin{aligned}
& R^{2}=1-\frac{\sum_{i=1}^{12}\left(\mathrm{ET}_{\mathrm{FPM}_{i}}-\mathrm{ET}_{\mathrm{m}_{i}}\right)^{2}}{\sum_{i=1}^{12}\left(\mathrm{ET}_{\mathrm{FPM}_{i}}-\frac{\sum_{i=1}^{12} \mathrm{ET}_{\mathrm{FPM}_{i}}}{12}\right)^{2}} \\
& \mathrm{MBE}=\frac{\sum_{i=1}^{12}\left(\mathrm{ET}_{\mathrm{FPM}_{i}}-\mathrm{ET}_{\mathrm{m}_{i}}\right)}{12}
\end{aligned}
$$

in which, $i$ indicates month, $\mathrm{ET}_{\mathrm{FPM}}$ indicates the reference crop evapotranspiration calculated for FAO PenmanMonteith (FPM) model, $\mathrm{ET}_{\mathrm{m}}$ indicates the reference crop evapotranspiration calculated for mass transfer-based models, and MBE is mean bias error (MBE). Meanwhile, the map of the error calculated for each province has been presented.

\section{Results and discussion}

Comparison of the best methods for each province

Tables 3, 4 and 5 and Figs. 1, 2 and 3 compare the potential evapotranspiration using FPM with values estimated using the $T$-based formula, $T / \mathrm{RH}$-based formula, and $T / \mathrm{RH} / u$-based formula, respectively, for each province.

$T$-based formula, $T / \mathrm{RH}$-based formula, and $T / \mathrm{RH} / u$ based formula overestimated the FPM for 29, 26, 24 provinces, respectively. The overestimation of the reference evapotranspiration values was also found in the other researches (Martinez and Thepadia 2010; Valipour 2014o, p, q, r, s, t, u, v, w, x).

According to Tables 3, 4 and 5 and Figs. 1, 2 and 3, T/ $\mathrm{RH} / u$-based formula for SK and GO $\left(R^{2}=1.00\right)$ yielded

\begin{tabular}{|c|c|c|c|c|}
\hline Province & T-based formula & Symbol & $R^{2}$ & MBE \\
\hline CB & $\mathrm{ET}_{\mathrm{o}}=0.186 T+0.937$ & Eq. 1 & 0.89 & 0.00 \\
\hline EA & $\mathrm{ET}_{\mathrm{o}}=0.274 T+0.485$ & Eq. 2 & 0.93 & 0.02 \\
\hline WA & $\mathrm{ET}_{\mathrm{o}}=0.2 T+0.576$ & Eq. 3 & 0.90 & 0.01 \\
\hline AR & $\mathrm{ET}_{\mathrm{o}}=0.185 T+1.074$ & Eq. 4 & 0.88 & -0.01 \\
\hline ES & $\mathrm{ET}_{\mathrm{o}}=0.238 T+0.34$ & Eq. 5 & 0.90 & 0.00 \\
\hline IL & $\mathrm{ET}_{\mathrm{o}}=0.273 T-0.3$ & Eq. 6 & 0.93 & 0.02 \\
\hline BU & $\mathrm{ET}_{\mathrm{o}}=0.286 T-2.057$ & Eq. 7 & 0.87 & 0.01 \\
\hline TE & $\mathrm{ET}_{\mathrm{o}}=0.267 T-0.0169$ & Eq. 8 & 0.91 & 0.00 \\
\hline AL & $\mathrm{ET}_{\mathrm{o}}=0.248 T+0.183$ & Eq. 9 & 0.90 & 0.01 \\
\hline SK & $\mathrm{ET}_{\mathrm{o}}=0.323 T-0.524$ & Eq. 10 & 0.95 & 0.00 \\
\hline RK & $\mathrm{ET}_{\mathrm{o}}=0.274 T-0.233$ & Eq. 11 & 0.95 & 0.01 \\
\hline NK & $\mathrm{ET}_{\mathrm{o}}=0.259 T-0.029$ & Eq. 12 & 0.93 & 0.03 \\
\hline $\mathrm{KH}$ & $\mathrm{ET}_{\mathrm{o}}=0.352 T-2.99$ & Eq. 13 & 0.92 & 0.01 \\
\hline ZA & $\mathrm{ET}_{\mathrm{o}}=0.205 T+0.89$ & Eq. 14 & 0.91 & 0.01 \\
\hline SE & $\mathrm{ET}_{\mathrm{o}}=0.231 T-0.379$ & Eq. 15 & 0.92 & 0.03 \\
\hline SB & $\mathrm{ET}_{\mathrm{o}}=0.304 T-0.128$ & Eq. 16 & 0.94 & 0.01 \\
\hline FA & $\mathrm{ET}_{\mathrm{o}}=0.272 T-0.195$ & Eq. 17 & 0.93 & 0.01 \\
\hline QO & $\mathrm{ET}_{\mathrm{o}}=0.272 T-0.507$ & Eq. 18 & 0.91 & 0.02 \\
\hline $\mathrm{GH}$ & $\mathrm{ET}_{\mathrm{o}}=0.24 T+0.278$ & Eq. 19 & 0.92 & 0.02 \\
\hline $\mathrm{KO}$ & $\mathrm{ET}_{\mathrm{o}}=0.231 T+0.557$ & Eq. 20 & 0.93 & -0.01 \\
\hline $\mathrm{KE}$ & $\mathrm{ET}_{\mathrm{o}}=0.311 T+0.136$ & Eq. 21 & 0.96 & 0.02 \\
\hline KS & $\mathrm{ET}_{\mathrm{o}}=0.262 T+0.259$ & Eq. 22 & 0.95 & 0.01 \\
\hline KB & $\mathrm{ET}_{\mathrm{o}}=0.219 T+0.228$ & Eq. 23 & 0.91 & 0.01 \\
\hline GO & $\mathrm{ET}_{\mathrm{o}}=0.19 T-0.656$ & Eq. 24 & 0.86 & 0.00 \\
\hline GI & $\mathrm{ET}_{\mathrm{o}}=0.169 T-0.439$ & Eq. 25 & 0.82 & 0.00 \\
\hline LO & $\mathrm{ET}_{\mathrm{o}}=0.237 T-0.246$ & Eq. 26 & 0.93 & 0.00 \\
\hline MZ & $\mathrm{ET}_{\mathrm{o}}=0.178 T-0.472$ & Eq. 27 & 0.82 & 0.00 \\
\hline MA & $\mathrm{ET}_{\mathrm{o}}=0.202 T+0.635$ & Eq. 28 & 0.91 & 0.00 \\
\hline $\mathrm{HO}$ & $\mathrm{ET}_{\mathrm{o}}=0.272 T-2.149$ & Eq. 29 & 0.90 & 0.01 \\
\hline HA & $\mathrm{ET}_{\mathrm{o}}=0.199 T+0.91$ & Eq. 30 & 0.91 & 0.00 \\
\hline YA & $\mathrm{ET}_{\mathrm{o}}=0.277 T-0.153$ & Eq. 31 & 0.94 & 0.03 \\
\hline
\end{tabular}

Table 3 Error of $T$-based formula for each province

$\mathrm{ET}_{\mathrm{o}}$ is the reference crop evapotranspiration ( $\mathrm{mm} /$ day) and $T$ is the average daily air temperature $\left({ }^{\circ} \mathrm{C}\right)$

the best potential evapotranspiration as compared to that from the FPM. However, if we do not access to wind speed and relative humidity, $T / \mathrm{RH}$-based formula and $T$-based formula, respectively, are very useful.

Determining range of weather parameters for the best methods to use for next studies

The maps of annual average of weather parameters (Fig. 4) are useful not only for the mentioned categories but also for determining the range of each parameter for which the best preciseness of the new methods is obtained (Table 6). 
This underlines the important role of selection of the best model for a specified weather conditions. Therefore, we can use the new formulas for other regions (in other countries) based on Table 6 with respect to their errors. The best weather conditions to use the formulas are 14-26 ${ }^{\circ} \mathrm{C}$ and $2.50-3.50 \mathrm{~m} / \mathrm{s}$ for temperature and wind speed, respectively. The results are also useful for selecting the best model when we must apply these formulas because of limitation of available data.
Comparison of the best methods with their errors for each province

Figure 4 was plotted to compare the error of the provinces. Although $R^{2}$ is more than 0.93 south east of Iran, it is the least value for categories I, II, III, and IV. This confirms that the categories are reliable and these four categories need more attention due to specific weather conditions. Thus, we need radiation, temperature, mass transfer, and

Table 4 Error of $T / \mathrm{RH}$-based formula for each province

\begin{tabular}{|c|c|c|c|c|}
\hline Province & $T /$ RH-based formula & Symbol & $R^{2}$ & MBE \\
\hline $\mathrm{CB}$ & $\mathrm{ET}_{\mathrm{o}}=0.367 T+0.12 \mathrm{RH}-6.851$ & Eq. 1 & 0.90 & 0.05 \\
\hline EA & $\mathrm{ET}_{\mathrm{o}}=0.177 T 5-0.071 \mathrm{RH}+5.545$ & Eq. 2 & 0.93 & 0.01 \\
\hline WA & $\mathrm{ET}_{\mathrm{o}}=0.115 T-0.0753 \mathrm{RH}+6.13$ & Eq. 3 & 0.91 & 0.01 \\
\hline AR & $\mathrm{ET}_{\mathrm{o}}=0.146 T-0.169 \mathrm{RH}+13.58$ & Eq. 4 & 0.94 & -0.05 \\
\hline ES & $\mathrm{ET}_{\mathrm{o}}=0.156 T-0.0594 \mathrm{RH}+4.042$ & Eq. 5 & 0.90 & 0.00 \\
\hline IL & $\mathrm{ET}_{\mathrm{o}}=0.377 T+0.053 \mathrm{RH}-4.186$ & Eq. 6 & 0.93 & 0.02 \\
\hline BU & $\mathrm{ET}_{\mathrm{o}}=0.178 T-0.151 \mathrm{RH}+10.482$ & Eq. 7 & 0.94 & -0.03 \\
\hline TE & $\mathrm{ET}_{\mathrm{o}}=0.244 T-0.0154 \mathrm{RH}+1.005$ & Eq. 8 & 0.91 & 0.00 \\
\hline AL & $\mathrm{ET}_{\mathrm{o}}=0.226 T-0.016 \mathrm{RH}+1.276$ & Eq. 9 & 0.90 & 0.01 \\
\hline SK & $\mathrm{ET}_{\mathrm{o}}=0.589 T+0.166 \mathrm{RH}-10.971$ & Eq. 10 & 0.99 & 0.01 \\
\hline RK & $\mathrm{ET}_{\mathrm{o}}=0.355 T+0.0449 \mathrm{RH}-3.853$ & Eq. 11 & 0.96 & 0.01 \\
\hline NK & $\mathrm{ET}_{\mathrm{o}}=0.287 T+0.0224 \mathrm{RH}-1.731$ & Eq. 12 & 0.93 & 0.03 \\
\hline KH & $\mathrm{ET}_{\mathrm{o}}=0.242 T-0.0584 \mathrm{RH}+2.31$ & Eq. 13 & 0.92 & 0.02 \\
\hline $\mathrm{ZA}$ & $\mathrm{ET}_{\mathrm{o}}=0.29 T+0.0644 \mathrm{RH}-3.559$ & Eq. 14 & 0.91 & 0.01 \\
\hline SE & $\mathrm{ET}_{\mathrm{o}}=0.196 T-0.0275 \mathrm{RH}+1.399$ & Eq. 15 & 0.93 & 0.02 \\
\hline SB & $\mathrm{ET}_{\mathrm{o}}=0.583 T+0.184 \mathrm{RH}-11.462$ & Eq. 16 & 1.00 & 0.03 \\
\hline FA & $\mathrm{ET}_{\mathrm{o}}=0.373 T+0.0558 \mathrm{RH}-4.278$ & Eq. 17 & 0.93 & 0.01 \\
\hline QO & $\mathrm{ET}_{\mathrm{o}}=0.452 T+0.112 \mathrm{RH}-8.408$ & Eq. 18 & 0.92 & 0.03 \\
\hline $\mathrm{GH}$ & $\mathrm{ET}_{\mathrm{o}}=0.346 T+0.0821 \mathrm{RH}-5.4$ & Eq. 19 & 0.93 & 0.02 \\
\hline $\mathrm{KO}$ & $\mathrm{ET}_{\mathrm{o}}=0.32 T+0.0495 \mathrm{RH}-3.013$ & Eq. 20 & 0.93 & -0.01 \\
\hline $\mathrm{KE}$ & $\mathrm{ET}_{\mathrm{o}}=0.565 T+0.167 \mathrm{RH}-9.374$ & Eq. 21 & 0.99 & 0.04 \\
\hline $\mathrm{KS}$ & $E T_{o}=0.38 T+0.0561 R H-4.104$ & Eq. 22 & 0.95 & 0.00 \\
\hline $\mathrm{KB}$ & $\mathrm{ET}_{\mathrm{o}}=0.422 T+0.103 \mathrm{RH}-7.483$ & Eq. 23 & 0.94 & 0.01 \\
\hline GO & $\mathrm{ET}_{\mathrm{o}}=0.0994 T-0.216 \mathrm{RH}+16.168$ & Eq. 24 & 0.91 & -0.06 \\
\hline GI & $\mathrm{ET}_{\mathrm{o}}=0.12 T-0.12 \mathrm{RH}+10.341$ & Eq. 25 & 0.96 & -0.03 \\
\hline LO & $\mathrm{ET}_{\mathrm{o}}=0.371 T+0.0686 \mathrm{RH}-5.761$ & Eq. 26 & 0.94 & 0.00 \\
\hline MZ & $\mathrm{ET}_{\mathrm{o}}=0.111 T-0.308 \mathrm{RH}+24.82$ & Eq. 27 & 0.97 & 0.00 \\
\hline MA & $\mathrm{ET}_{\mathrm{o}}=0.308 T+0.0631 \mathrm{RH}-3.775$ & Eq. 28 & 0.91 & 0.00 \\
\hline $\mathrm{HO}$ & $\mathrm{ET}_{\mathrm{o}}=0.276 T-0.0527 \mathrm{RH}+1.174$ & Eq. 29 & 0.91 & 0.01 \\
\hline HA & $\mathrm{ET}_{\mathrm{o}}=0.372 T+0.105 \mathrm{RH}-6.734$ & Eq. 30 & 0.93 & 0.03 \\
\hline YA & $\mathrm{ET}_{\mathrm{o}}=0.482 T+0.151 \mathrm{RH}-8.896$ & Eq. 31 & 0.97 & 0.03 \\
\hline
\end{tabular}

$\mathrm{ET}_{\mathrm{o}}$ is the reference crop evapotranspiration ( $\mathrm{mm} /$ day), $T$ is the average daily air temperature $\left({ }^{\circ} \mathrm{C}\right)$, and $\mathrm{RH}$ is the average relative humidity $(\%)$ 
pan evaporation-based models to estimate the reference crop evapotranspiration in this province. It reveals that only if we use the new methods for suitable (based on Table 6) and specific (based on Fig. 4) weather conditions, the highest preciseness of estimating will be obtained.

More accurate estimation of potential evapotranspiration can help to other studies including agricultural water management (Valipour 2012a, b, c, d, e, f, g, h, i, j, 2013a, b, c, d, e, f, g, h, 2014), and water resources management (Banihabib et al. 2012; Valipour et al. 2012a, b, c, d, 2013a, b, c; Valipour and Montazar 2012a, b, c).

\section{Conclusion}

Although, the average value of weather parameters in a certain province is used for evapotranspiration estimation of that province, the evapotranspiration is a function of many weather parameters and a significant underestimation or overestimation of evapotranspiration for a province occurs for considerable variations of weather parameters. Therefore, possibility of simultaneous difference of some weather parameters with their average values leads to a significant underestimation or overestimation of

Table 5 Error of $T / \mathrm{RH} / u$-based formula for each province

\begin{tabular}{|c|c|c|c|c|}
\hline Province & $T / \mathrm{RH} / u$-based formula & Symbol & $R^{2}$ & MBE \\
\hline $\mathrm{CB}$ & $\mathrm{ET}_{\mathrm{o}}=0.317 T+0.0911 \mathrm{RH}+0.866 u-5.893$ & Eq. 1 & 0.95 & 0.01 \\
\hline EA & $\mathrm{ET}_{\mathrm{o}}=0.173 T+0.0284 \mathrm{RH}+1.639 u-4.889$ & Eq. 2 & 0.99 & 0.02 \\
\hline WA & $\mathrm{ET}_{\mathrm{o}}=0.169 T-0.00687 \mathrm{RH}+1.105 u-0.135$ & Eq. 3 & 0.93 & 0.00 \\
\hline AR & $\mathrm{ET}_{\mathrm{o}}=0.168 T-0.0853 \mathrm{RH}+0.721 u+4.589$ & Eq. 4 & 0.95 & -0.02 \\
\hline ES & $\mathrm{ET}_{\mathrm{o}}=0.332 T+0.0874 \mathrm{RH}+1.203 u-7.166$ & Eq. 5 & 0.98 & -0.01 \\
\hline IL & $\mathrm{ET}_{\mathrm{o}}=0.321 T+0.0666 \mathrm{RH}+2.153 u-8.393$ & Eq. 6 & 0.98 & 0.01 \\
\hline $\mathrm{BU}$ & $\mathrm{ET}_{\mathrm{o}}=0.299 T+0.0162 \mathrm{RH}+1.615 u-8.365$ & Eq. 7 & 1.00 & 0.01 \\
\hline $\mathrm{TE}$ & $\mathrm{ET}_{\mathrm{o}}=0.457 T+0.157 \mathrm{RH}+1.474 u-13.719$ & Eq. 8 & 0.98 & 0.03 \\
\hline $\mathrm{AL}$ & $\mathrm{ET}_{\mathrm{o}}=0.369 T+0.118 \mathrm{RH}+1.754 u-11.316$ & Eq. 9 & 0.99 & 0.01 \\
\hline SK & $\mathrm{ET}_{\mathrm{o}}=0.409 T+0.0909 \mathrm{RH}+0.762 u-7.259$ & Eq. 10 & 1.00 & 0.00 \\
\hline RK & $\mathrm{ET}_{\mathrm{o}}=0.201 T-0.01 R H+1.096 u-0.951$ & Eq. 11 & 0.97 & 0.01 \\
\hline NK & $\mathrm{ET}_{\mathrm{o}}=0.104 T-0.0452 \mathrm{RH}+1.352 u+1.604$ & Eq. 12 & 0.99 & 0.01 \\
\hline $\mathrm{KH}$ & $\mathrm{ET}_{\mathrm{o}}=0.32 T+0.0452 \mathrm{RH}+2.334 u-10.143$ & Eq. 13 & 1.00 & 0.02 \\
\hline $\mathrm{ZA}$ & $\mathrm{ET}_{\mathrm{o}}=0.297 T+0.0772 \mathrm{RH}+1.787 u-7.736$ & Eq. 14 & 0.97 & 0.01 \\
\hline SE & $\mathrm{ET}_{\mathrm{o}}=0.327 T+0.195 \mathrm{RH}+3.674 u-15.206$ & Eq. 15 & 0.99 & 0.03 \\
\hline SB & $\mathrm{ET}_{\mathrm{o}}=0.489 T+0.121 \mathrm{RH}+0.311 u-8.646$ & Eq. 16 & 1.00 & 0.02 \\
\hline FA & $\mathrm{ET}_{\mathrm{o}}=0.308 T+0.0405 \mathrm{RH}+1.336 u-5.617$ & Eq. 17 & 0.99 & 0.02 \\
\hline QO & $\mathrm{ET}_{\mathrm{o}}=0.363 T+0.101 \mathrm{RH}+1.519 u-9.37$ & Eq. 18 & 0.99 & 0.03 \\
\hline GH & $\mathrm{ET}_{\mathrm{o}}=0.366 T+0.147 R H+1.701 u-12.419$ & Eq. 19 & 0.98 & 0.04 \\
\hline $\mathrm{KO}$ & $\mathrm{ET}_{\mathrm{o}}=0.267 T+0.0294 \mathrm{RH}+1.049 u-3.459$ & Eq. 20 & 0.96 & 0.00 \\
\hline $\mathrm{KE}$ & $\mathrm{ET}_{\mathrm{o}}=0.48 T+0.122 \mathrm{RH}+0.308 u-7.503$ & Eq. 21 & 1.00 & 0.02 \\
\hline $\mathrm{KS}$ & $\mathrm{ET}_{\mathrm{o}}=0.312 T+0.0285 \mathrm{RH}+0.959 u-4.229$ & Eq. 22 & 0.97 & 0.02 \\
\hline $\mathrm{KB}$ & $\mathrm{ET}_{\mathrm{o}}=0.312 T+0.0579 \mathrm{RH}+1.334 u-5.532$ & Eq. 23 & 0.97 & -0.02 \\
\hline GO & $\mathrm{ET}_{\mathrm{o}}=0.0933 T-0.0413 \mathrm{RH}+3.001 u+0.685$ & Eq. 24 & 1.00 & 0.00 \\
\hline GI & $\mathrm{ET}_{\mathrm{o}}=0.146 T-0.114 \mathrm{RH}+0.912 u+8.315$ & Eq. 25 & 0.96 & -0.07 \\
\hline LO & $\mathrm{ET}_{\mathrm{o}}=0.311 T+0.0447 \mathrm{RH}+1.385 u-5.899$ & Eq. 26 & 0.97 & -0.02 \\
\hline MZ & $\mathrm{ET}_{\mathrm{o}}=0.124 T-0.217 \mathrm{RH}+1.139 u+15.297$ & Eq. 27 & 0.98 & -0.05 \\
\hline MA & $\mathrm{ET}_{\mathrm{o}}=0.336 T+0.0897 \mathrm{RH}+1.094 u-7.038$ & Eq. 28 & 0.96 & 0.00 \\
\hline $\mathrm{HO}$ & $\mathrm{ET}_{\mathrm{o}}=0.147 T-0.156 \mathrm{RH}+2.232 u+4.912$ & Eq. 29 & 0.97 & 0.00 \\
\hline HA & $\mathrm{ET}_{\mathrm{o}}=0.35 T+0.0944 \mathrm{RH}+0.625 u-6.9$ & Eq. 30 & 0.96 & -0.01 \\
\hline YA & $\mathrm{ET}_{\mathrm{o}}=0.33 T+0.0657 \mathrm{RH}+1.299 u-6.621$ & Eq. 31 & 1.00 & 0.01 \\
\hline
\end{tabular}

$\mathrm{ET}_{\mathrm{o}}$ is the reference crop evapotranspiration (mm/day), $T$ is the average daily air temperature $\left({ }^{\circ} \mathrm{C}\right), u$ is the mean daily wind speed at $2 \mathrm{~m}(\mathrm{~m} / \mathrm{s})$, and $\mathrm{RH}$ is the average relative humidity (\%) 


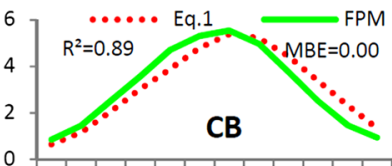

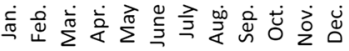

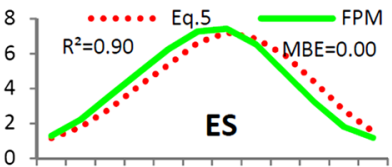

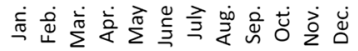
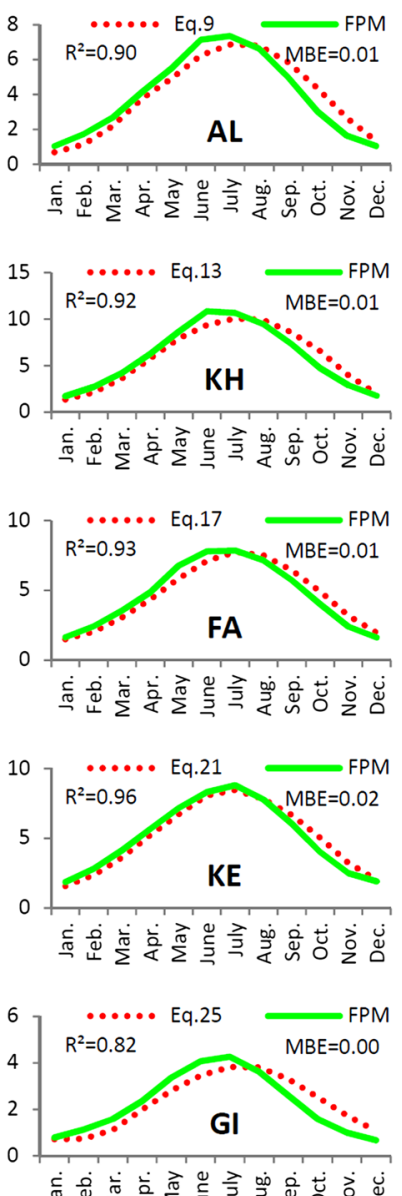

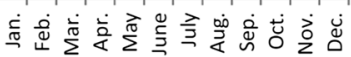

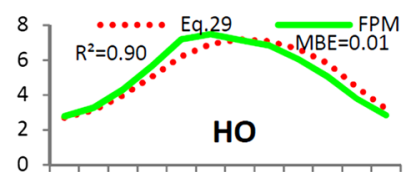

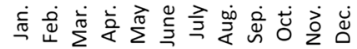

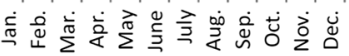

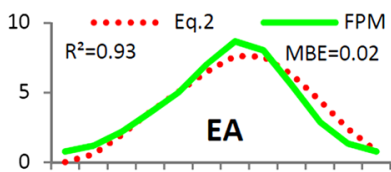

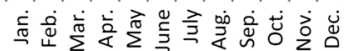
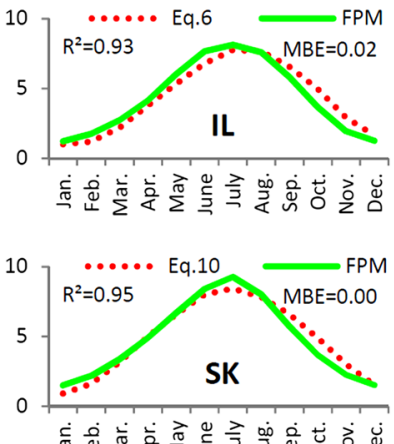

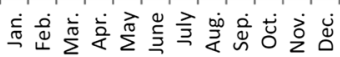
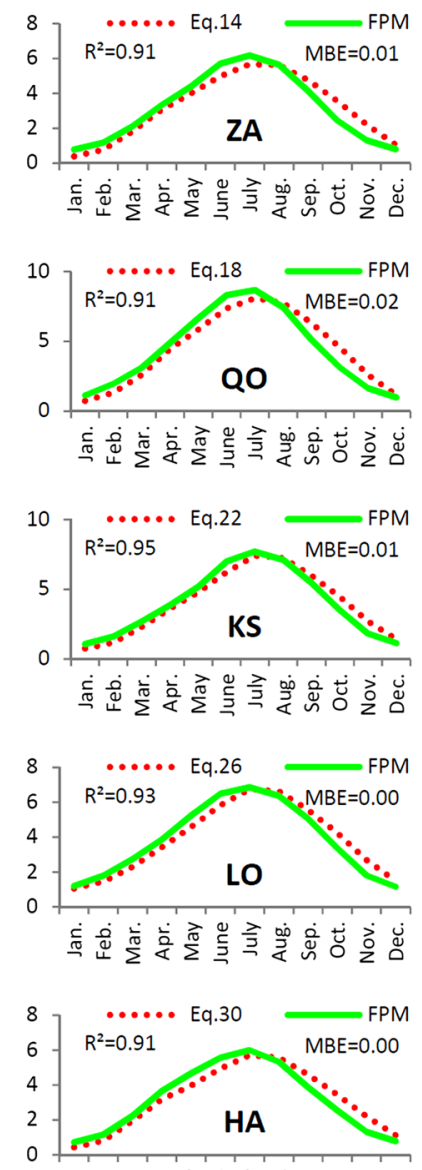

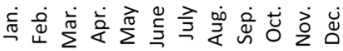

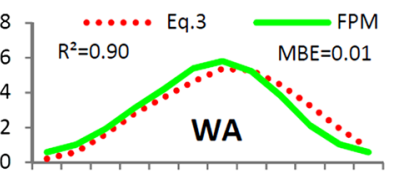

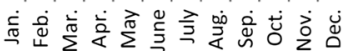
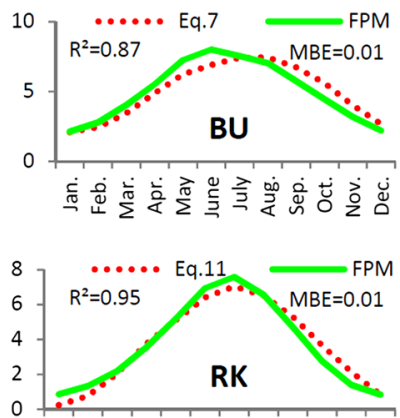

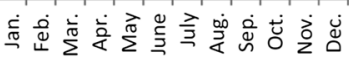
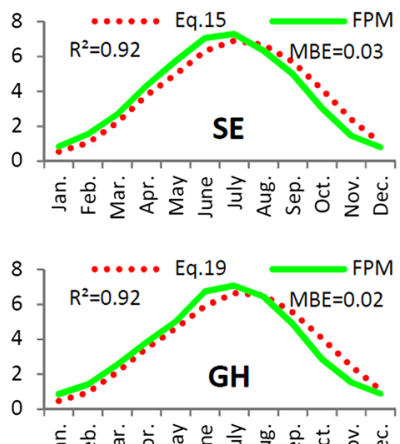

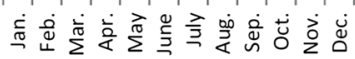
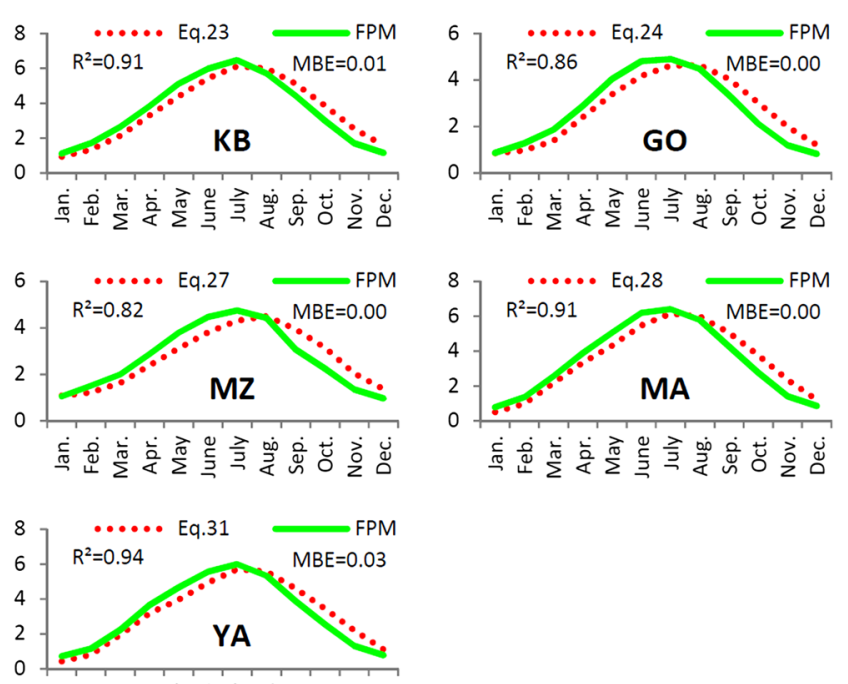

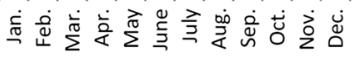

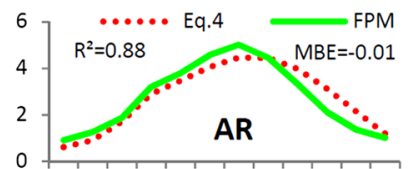

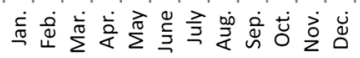
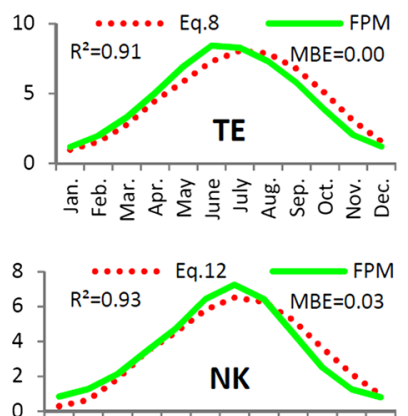

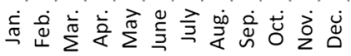
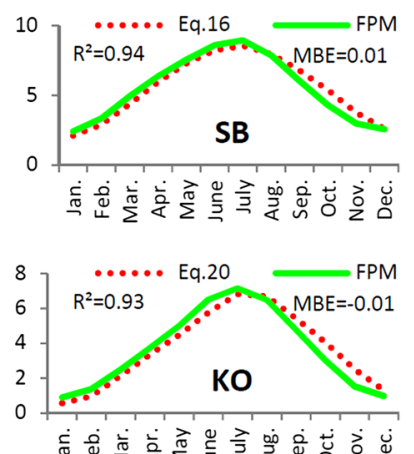

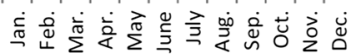

Fig. 1 Comparison of evapotranspiration calculated using FAO Penman-Monteith (FPM) with values estimated using the $T$-based formula 


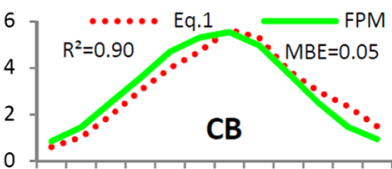

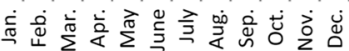

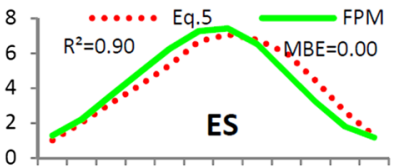

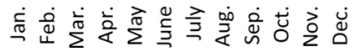

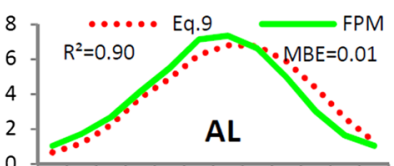

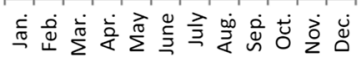
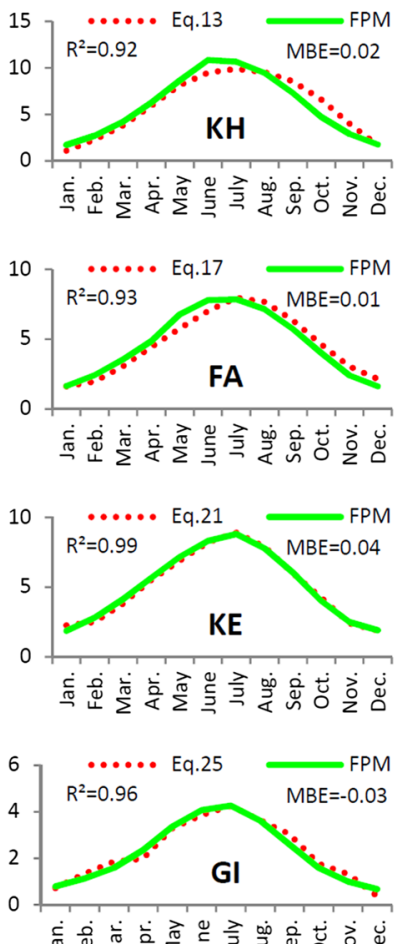

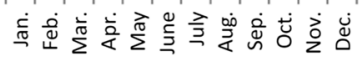

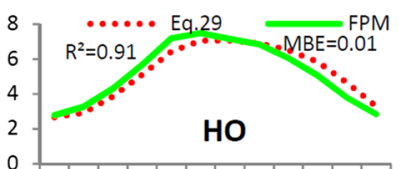

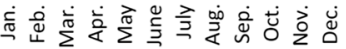

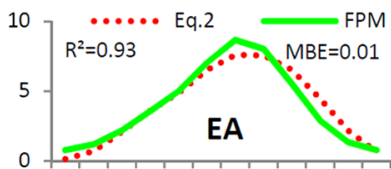

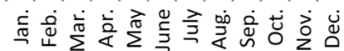
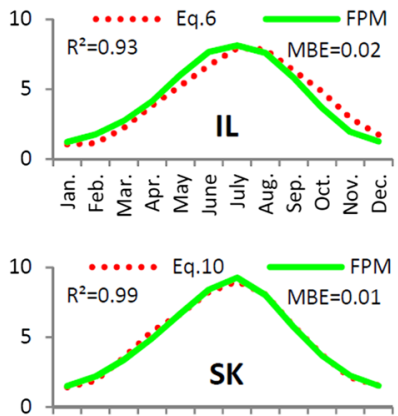

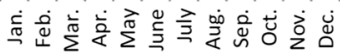
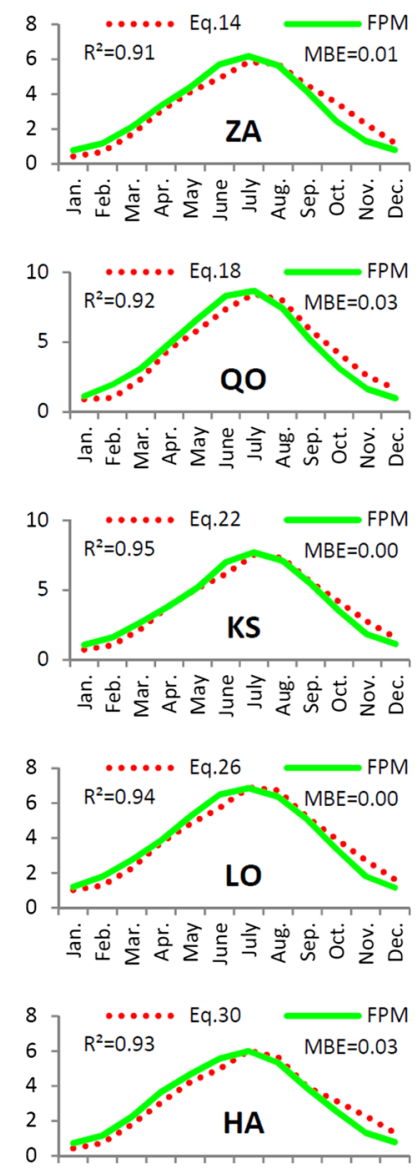

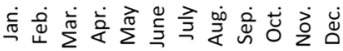

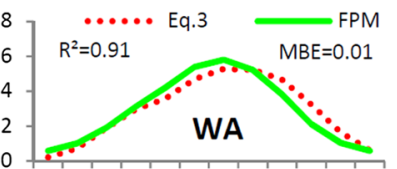

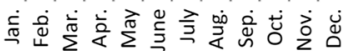
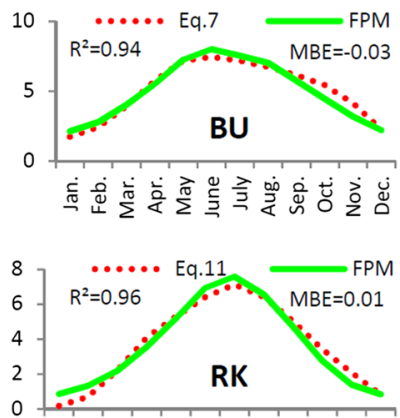

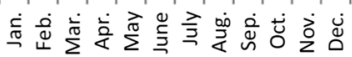
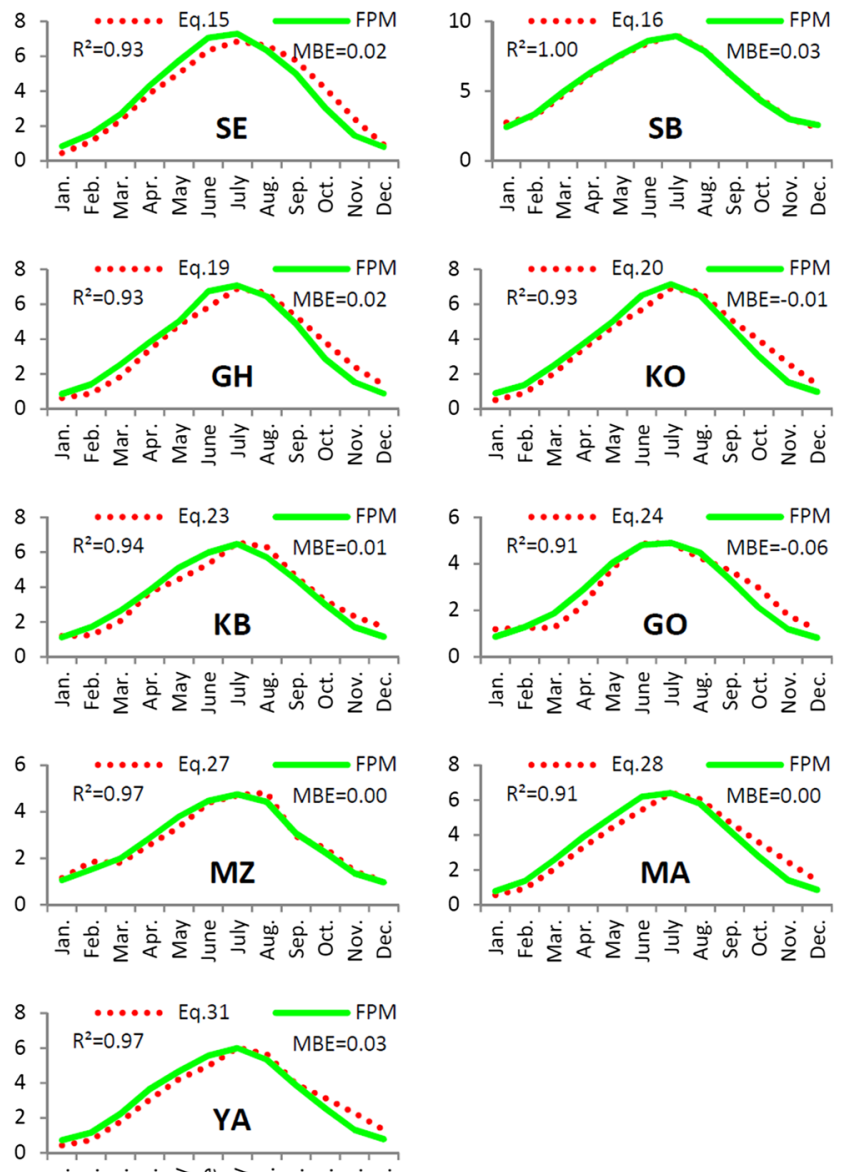

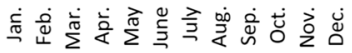

Fig. 2 Comparison of evapotranspiration calculated using FAO Penman-Monteith (FPM) potential evapotranspiration using FPM with values estimated using the $T / \mathrm{RH}$-based formula 


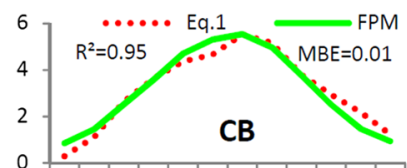

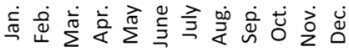

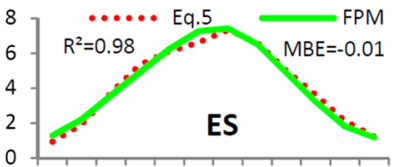

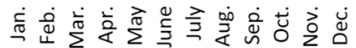

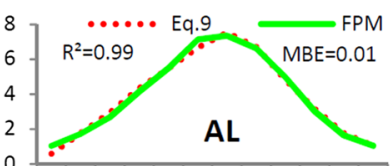

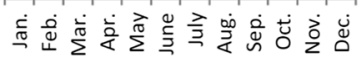
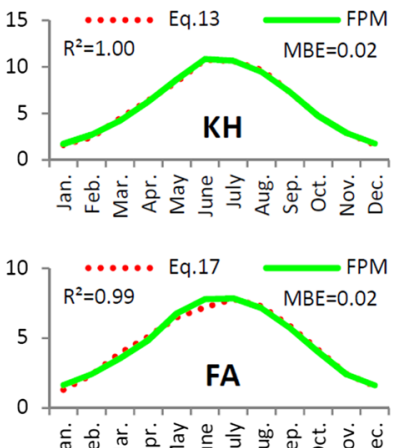

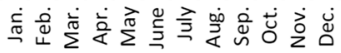
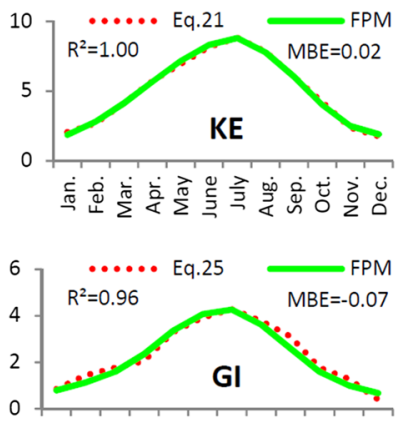

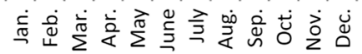

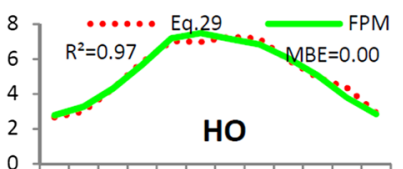

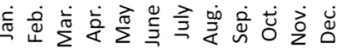

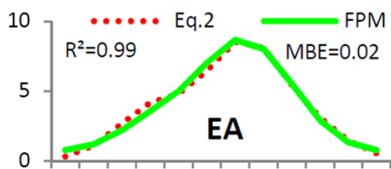

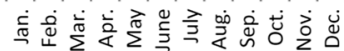
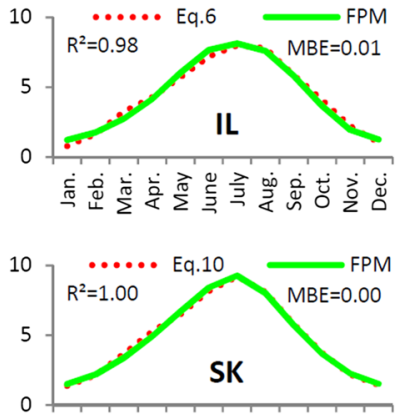

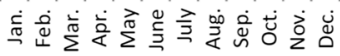
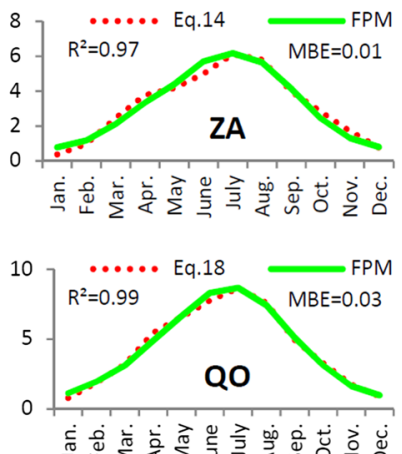

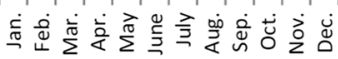
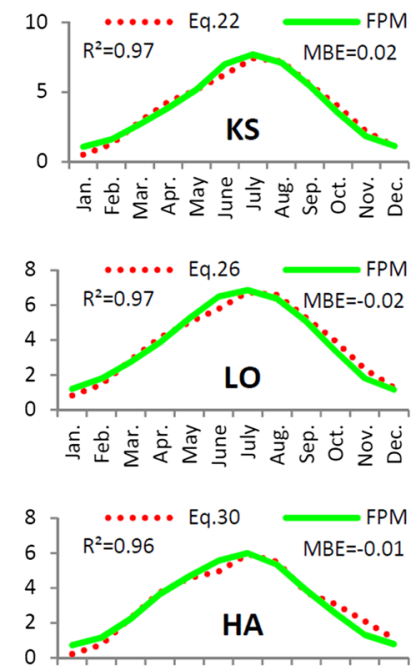

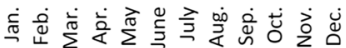

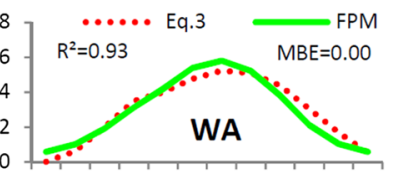

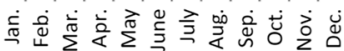
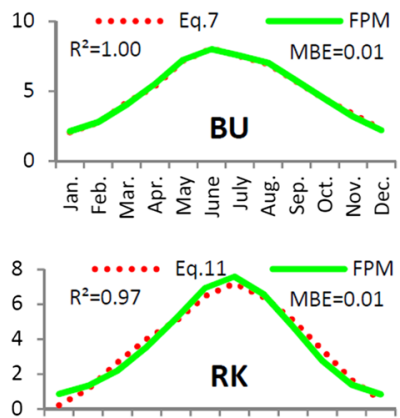

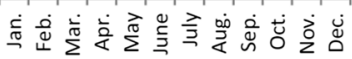
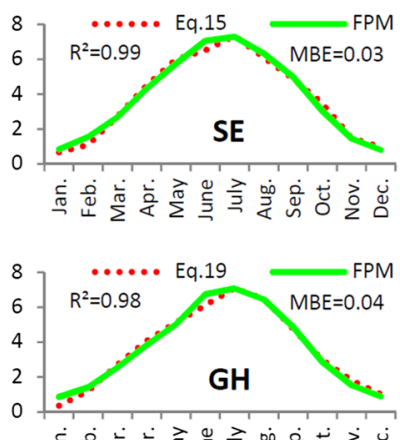

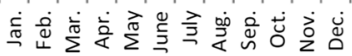
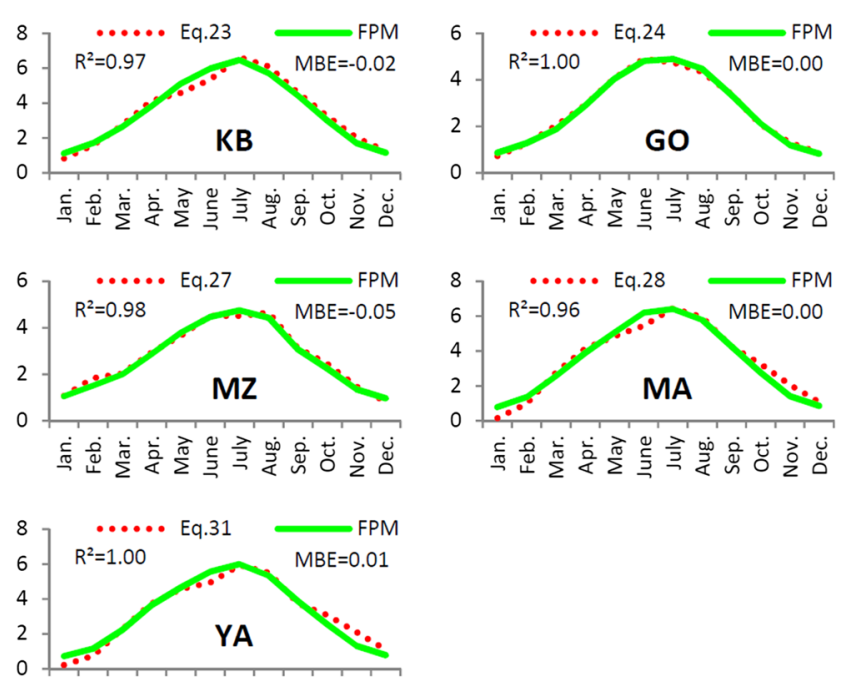

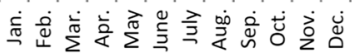

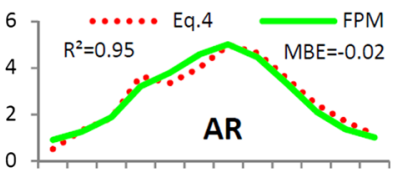

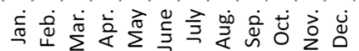
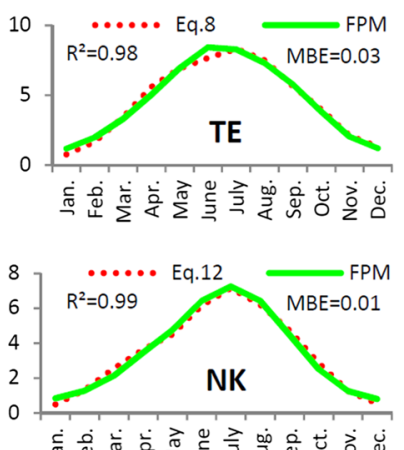

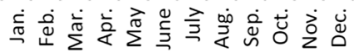
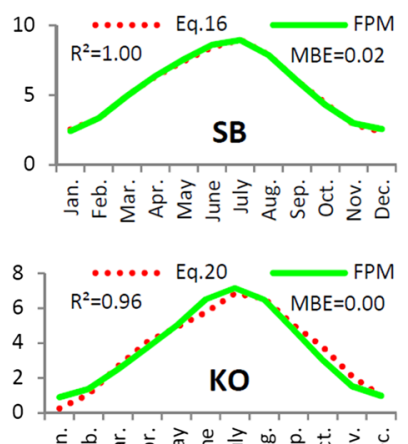

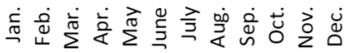

Fig. 3 Comparison of evapotranspiration calculated using FAO Penman-Monteith (FPM) potential evapotranspiration using FPM with values estimated using $T / \mathrm{RH} / u$-based formula 

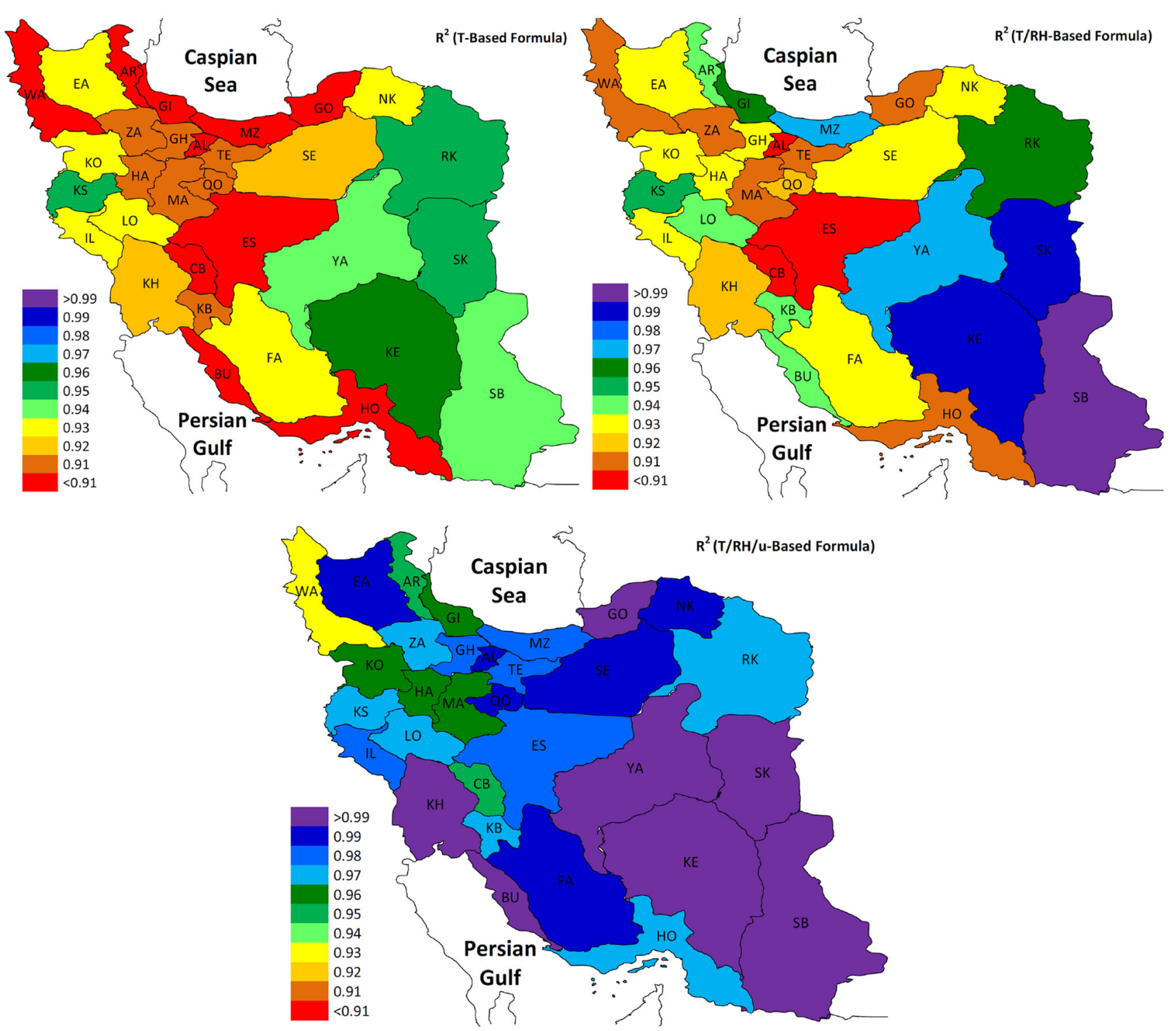

Fig. 4 Error of $T$-based, $T / \mathrm{RH}$-based, and $T / \mathrm{RH} / u$-based formulae

evapotranspiration for a province is poor. However, it is better to spatially distribute the weather parameters first, then to estimate the water requirements for each province for better estimation of crop water requirement of each province. In a study by Basharat and Tariq (2013), for example, they observed that the tail reaches require $33 \%$ (maximum) more water than the head reaches due to variation of rainfall in LBDC canal command in Pakistan. Also in some studies the Penman-Monteith method shows the $10 \%$ variation when compared with the lysimeter data. Therefore, replacement of FPM model with lysimeter data can be recommended for next studies (Valipour 2014j). 
Table 6 The best range to use $T / \mathrm{RH} / u$-based formula based on the results of the current study

\begin{tabular}{|c|c|c|c|c|c|}
\hline Model & $T$ & RH & $u$ & $R^{2}$ & MBE \\
\hline Eq. 1 & $10-12$ & $45-50$ & $<1.25$ & 0.95 & 0.01 \\
\hline Eq. 2 & $12-14$ & $50-55$ & $3.00-3.25$ & 0.99 & 0.02 \\
\hline Eq. 3 & $10-12$ & $60-65$ & $1.25-1.50$ & 0.93 & 0.00 \\
\hline Eq. 4 & $8-10$ & $70-75$ & $>3.50$ & 0.95 & -0.02 \\
\hline Eq. 5 & $16-18$ & $35-40$ & $2.00-2.25$ & 0.98 & -0.01 \\
\hline Eq. 6 & $16-18$ & $40-45$ & $2.00-2.25$ & 0.98 & 0.01 \\
\hline Eq. 7 & $24-26$ & $65-70$ & $3.00-3.25$ & 1.00 & 0.01 \\
\hline Eq. 8 & $16-18$ & $40-45$ & $2.50-2.75$ & 0.98 & 0.03 \\
\hline Eq. 9 & $14-16$ & $45-50$ & $2.25-2.50$ & 0.99 & 0.01 \\
\hline Eq. 10 & $16-18$ & $35-40$ & $2.50-2.75$ & 1.00 & 0.00 \\
\hline Eq. 11 & $14-16$ & $55-60$ & $2.00-2.25$ & 0.97 & 0.01 \\
\hline Eq. 12 & $12-14$ & $55-60$ & $2.25-2.50$ & 0.99 & 0.01 \\
\hline Eq. 13 & $24-26$ & $40-45$ & $2.50-2.75$ & 1.00 & 0.02 \\
\hline Eq. 14 & $10-12$ & $50-55$ & $1.75-2.00$ & 0.97 & 0.01 \\
\hline Eq. 15 & $18-20$ & $40-45$ & $1.25-1.50$ & 0.99 & 0.03 \\
\hline Eq. 16 & $18-20$ & $<35$ & $3.25-3.50$ & 1.00 & 0.02 \\
\hline Eq. 17 & $16-18$ & $40-45$ & $2.25-2.50$ & 0.99 & 0.02 \\
\hline Eq. 18 & $16-18$ & $40-45$ & $1.75-2.00$ & 0.99 & 0.03 \\
\hline Eq. 19 & $12-14$ & $50-55$ & $2.00-2.25$ & 0.98 & 0.04 \\
\hline Eq. 20 & $12-14$ & $45-50$ & $2.00-2.25$ & 0.96 & 0.00 \\
\hline Eq. 21 & $14-16$ & $<35$ & $3.00-3.25$ & 1.00 & 0.02 \\
\hline Eq. 22 & $14-16$ & $45-50$ & $2.25-2.50$ & 0.97 & 0.02 \\
\hline Eq. 23 & $14-16$ & $40-45$ & $1.25-1.50$ & 0.97 & -0.02 \\
\hline Eq. 24 & $16-18$ & $70-75$ & $<1.25$ & 1.00 & 0.00 \\
\hline Eq. 25 & $14-16$ & $>80$ & $1.25-1.50$ & 0.96 & -0.07 \\
\hline Eq. 25 & $16-18$ & $45-50$ & $1.50-1.75$ & 0.97 & -0.02 \\
\hline Eq. 27 & $16-18$ & $75-80$ & $1.75-2.00$ & 0.98 & -0.05 \\
\hline Eq. 27 & $12-14$ & $45-50$ & $1.25-1.50$ & 0.96 & 0.00 \\
\hline Eq. 29 & $>26$ & $65-70$ & $2.75-3.00$ & 0.97 & 0.00 \\
\hline Eq. 30 & $10-12$ & $50-55$ & $1.50-1.75$ & 0.96 & -0.01 \\
\hline Eq. 31 & $18-20$ & $<35$ & $2.50-2.75$ & 1.00 & 0.0 \\
\hline
\end{tabular}

$T$ is the average daily air temperature $\left({ }^{\circ} \mathrm{C}\right), u$ is the mean daily wind speed at $2 \mathrm{~m}(\mathrm{~m} / \mathrm{s})$, and $\mathrm{RH}$ is the average relative humidity (\%)

Open Access This article is distributed under the terms of the Creative Commons Attribution License which permits any use, distribution, and reproduction in any medium, provided the original author(s) and the source are credited.

\section{References}

Al-Ghobari HM (2000) Estimation of potential evapotranspiration for southern region of Saudi Arabia. Irrig Sci 19(2):81-86

Allen RG, Pereira LS, Raes D, Smith M (1998) Crop evapotranspiration. Guidelines for computing crop water requirements. FAO Irrigation and Drainage. Paper no. 56, FAO, Rome

Banihabib ME, Valipour M, Behbahani SMR (2012) Comparison of autoregressive static and artificial dynamic neural network for the forecasting of monthly inflow of Dez reservoir. J Environ Sci Technol 13:1-14. http://jest.srbiau.ac.ir/?_action=articleInfo\&article=84
Basharat M, Tariq AuR (2013) Spatial climate variability and its impact on irrigated hydrology in a canal command. Arab J Sci Eng 38(3):507-522

Jacobs AFG, Heusinkveld BG, Holtslag AAM (2010) Eighty years of meteorological observations at Wageningen, the Netherlands: precipitation and evapotranspiration. Int J Climatol 30:1315-1321

Li L, Luo G, Chen X, Li Y, Xu G, Xu H, Bai J (2011) Modelling evapotranspiration in a Central Asian desert ecosystem. Ecol Model 222(20):3680-3691

Martinez C, Thepadia M (2010) Estimating potential evapotranspiration with minimum data in Florida. J Irrig Drain Eng 136(7):494-501

Rahimi S, Gholami Sefidkouhi MA, Raeini-Sarjaz M, Valipour M (2014) Estimation of actual evapotranspiration by using MODIS images (a case study: Tajan catchment). Arch Agron Soil Sci. doi: $10.1080 / 03650340.2014 .944904$

Rojas J, Sheffield R (2013) Evaluation of daily potential evapotranspiration methods as compared with the ASCE-EWRI PenmanMonteith equation using limited weather data in Northeast Louisiana. J Irrig Drain Eng 139(4):285-292

Shi T, Guan D, Wang A, Wu J, Jin C, Han S (2008) Comparison of three models to estimate evapotranspiration for a temperate mixed forest. Hydrol Process 22:3431-3443

Valipour M (2012a) Critical areas of Iran for agriculture water management according to the annual rainfall. Eur J Sci Res 84:600-608

Valipour M (2012b) Comparison of surface irrigation simulation models: full hydrodynamic, zero inertia, kinematic wave. J Agr Sci $4: 68-74$

Valipour M (2012c) Determining possible optimal values of required flow, nozzle diameter, and wetted area for linear traveling laterals. Int J Eng Sci 1(1):37-43. http://www.theijes.com/ papers/v1-i1/H011037043.pdf

Valipour M (2012d) Hydro-module determination for Vanaei Village in Eslam Abad Gharb, Iran. ARPN J Agric Biol Sci 7:968-976

Valipour M (2012e) Number of required observation data for rainfall forecasting according to the climate conditions. Am J Sci Res 74:79-86

Valipour M (2012f) Scrutiny of pressure loss, friction slope, inflow velocity, velocity head, and Reynolds number in center pivot. Int J Adv Sci Technol Res 2:703-711

Valipour M (2012g) Ability of box-Jenkins models to estimate of reference potential evapotranspiration (a case study: Mehrabad Synoptic Station, Tehran, Iran). IOSR J Agric Vet Sci 1:1-11

Valipour M (2012h) Effect of drainage parameters change on amount of drain discharge in subsurface drainage systems. IOSR J Agric Vet Sci 1:10-18

Valipour M (2012i) A comparison between horizontal and vertical drainage systems (include pipe drainage, open ditch drainage, and pumped wells) in anisotropic soils. IOSR J Mech Civil Eng $4: 7-12$

Valipour M (2012j) Sprinkle and trickle irrigation system design using tapered pipes for pressure loss adjusting. J Agric Sci 4(12):125-133

Valipour M (2013a) Necessity of irrigated and rainfed agriculture in the world. Irrig Drain Sys Eng S9:e001. http://omicsgroup.org/ journals/necessity-of-irrigated-and-rainfed-agriculture-in-theworld-2168-9768.S9-e001.php?aid=12800

Valipour M (2013b) Evolution of irrigation-equipped areas as share of cultivated areas. Irrig Drain Sys Eng 2:e114. doi:10.4172/2168$9768.1000 \mathrm{e} 114$

Valipour M (2013c) Need to update of irrigation and water resources information according to the progresses of agricultural knowledge. Agrotechnology S10:e001. doi:10.4172/2168-9881.S10-e001

Valipour M (2013d) Increasing irrigation efficiency by management strategies: cutback and surge irrigation. ARPN J Agric Biol Sci $8: 35-43$ 
Valipour M (2013e) Use of surface water supply index to assessing of water resources management in Colorado and Oregon, US. Adv Agric Sci Eng Res 3:631-640

Valipour M (2013f) Estimation of surface water supply index using snow water equivalent. Adv Agric Sci Eng Res 3:587-602

Valipour M (2013g) Scrutiny of inflow to the drains applicable for improvement of soil environmental conditions. In: The 1st international conference on environmental crises and its solutions, Kish Island. http://www.civilica.com/EnPaper-ICECS01ICECS01_048.html

Valipour M (2013h) Comparison of different drainage systems usable for solution of environmental crises in soil. In: The 1st international conference on environmental crises and its solutions, Kish Island. http://www.civilica.com/EnPaper-ICECS01ICECS01_047.html

Valipour M (2014a) Importance of solar radiation, temperature, relative humidity, and wind speed for calculation of reference evapotranspiration. Arch Agron Soil Sci. doi:10.1080/03650340.2014.925107

Valipour M (2014b) Handbook of water engineering problems. OMICS Group eBooks, Foster City. http://www.esciencecentral. org/ebooks/handbook-of-water-engineering-problems/pdf/hand book-of-water-engineering-problems.pdf

Valipour M (2014c) Future of the area equipped for irrigation. Arch Agron Soil Sci 60(12):1641-1660

Valipour M (2014d) Temperature analysis of reference evapotranspiration models. Meteorol Appl. doi:10.1002/met.1465

Valipour M (2014e) Application of new mass transfer formulae for computation of evapotranspiration. J Appl Water Eng Res 2(1):33-46

Valipour M (2014f) Land use policy and agricultural water management of the previous half of century in Africa. Appl Water Sci. doi:10.1007/s13201-014-0199-1

Valipour M (2014g) Future of agricultural water management in Americas. J Agric Res 54(2):245-268

Valipour M (2014h) Drainage, waterlogging, and salinity. Arch Agron Soil Sci 60(12):1625-1640

Valipour M (2014i) Future of agricultural water management in Europe based on socioeconomic indices. Acta Adv Agric Sci 2(7):1-18

Valipour M (2014j) Use of average data of 181 synoptic stations for estimation of reference crop evapotranspiration by temperaturebased methods. Water Resour Manag 28(12):4237-4255

Valipour M (2014k) Investigation of Valiantzas' evapotranspiration equation in Iran. Theor Appl Climatol. doi:10.1007/s00704-014-1240-x

Valipour M (2014l) Assessment of different equations to estimate potential evapotranspiration versus FAO Penman Monteith method. Acta Adv Agric Sci 2(11) (Accepted)

Valipour M (2014m) Future of agricultural water management in Africa. Arch Agron Soil Sci. doi:10.1080/03650340.2014.961433

Valipour M (2014n) Runoff long term study using SARIMA and ARIMA models in the United States. Meteorol Appl, Accepted

Valipour M (2014o) Comparative evaluation of radiation-based methods for estimation of reference evapotranspiration. J Hydrol Eng. doi:10.1061/(ASCE)HE.1943-5584.0001066

Valipour M (2014p) Handbook of irrigation engineering problems. OMICS Group eBooks, Foster City. http://www.esciencecentral. org/ebooks/handbook-of-irrigation-engineering-problems/pdf/ handbook-of-irrigation-engineering-problems.pdf

Valipour M (2014q) Handbook of hydraulic engineering problems. OMICS Group eBooks, Foster City. http://www.esciencecentral. org/ebooks/handbook-of-hydraulic-engineering-problems/pdf/ handbook-of-hydraulic-engineering-problems.pdf

Valipour M (2014r) Pressure on renewable water resources by irrigation to 2060. Acta Adv Agric Sci 2(8):32-42

Valipour M (2014s) Prediction of irrigated agriculture in Asia Pacific using FAO indices. Acta Adv Agric Sci 2(9):40-53

Valipour M (2014t) Handbook of environmental engineering problems. OMICS Group eBooks, Foster City. http://www.escience central.org/ebooks/handbook-of-environmental-engineering-probl ems/pdf/handbook-of-environmental-engineering-problems.pdf

Valipour M (2014u) Handbook of drainage engineering problems. OMICS Group eBooks, Foster City. http://www.esciencecentral. org/ebooks/handbook-of-drainage-engineering-problems/pdf/hand book-of-drainage-engineering-problems.pdf

Valipour M (2014v) Study of different climatic conditions to assess the role of solar radiation in reference crop evapotranspiration equations. Arch Agron Soil Sci. doi:10.1080/03650340.2014.941823

Valipour M (2014w) Handbook of hydrologic engineering problems. OMICS Group eBooks, Foster City. http://www.esciencecentral. org/ebooks/handbook-of-hydrologic-engineering-problems/pdf/ handbook-of-hydrologic-engineering-problems.pdf

Valipour M (2014x) Future of agricultural water management in Africa. Arch Agron Soil Sci. doi:10.1080/03650340.2014. 961433

Valipour M, Eslamian S (2014) Analysis of potential evapotranspiration using 11 modified temperature-based models. Int J Hydrol Sci Technol 4(3) (Accepted)

Valipour M, Montazar AA (2012a) Optimize of all effective infiltration parameters in furrow irrigation using visual basic and genetic algorithm programming. Aust J Basic Appl Sci 6:132-137

Valipour M, Montazar AA (2012b) Sensitive analysis of optimized infiltration parameters in SWDC model. Adv Environ Biol 6:2574-2581

Valipour M, Montazar AA (2012c) An evaluation of SWDC and WINSRFR models to optimize of infiltration parameters in furrow irrigation. Am J Sci Res 69:128-142

Valipour M, Banihabib ME, Behbahani SMR (2012a) Parameters estimate of autoregressive moving average and autoregressive integrated moving average models and compare their ability for inflow forecasting. J Math Stat 8(3):330-338

Valipour M, Banihabib ME, Behbahani SMR (2012b) Monthly inflow forecasting using autoregressive artificial neural network. J Appl Sci 12(20):2139-2147

Valipour M, Mousavi SM, Valipour R, Rezaei E (2012c) Air, water, and soil pollution study in industrial units using environmental flow diagram. J Basic Appl Sci Res 2:12365-12372

Valipour M, Mousavi SM, Valipour R, Rezaei E (2012d) SHCP: soil heat calculator program. IOSR J Appl Phys 2:44-50

Valipour M, Banihabib ME, Behbahani SMR (2013a) Comparison of the ARMA, ARIMA, and the autoregressive artificial neural network models in forecasting the monthly inflow of Dez dam reservoir. J Hydrol 476:433-441

Valipour M, Mousavi SM, Valipour R, Rezaei E (2013b) Deal with environmental challenges in civil and energy engineering projects using a new technology. J Civil Environ Eng 3:127

Valipour M, Mousavi SM, Valipour R, Rezaei E (2013b) A new approach for environmental crises and its solutions by computer modeling. In: The 1st international conference on environmental crises and its solutions, Kish Island. http://www.civilica.com/ EnPaper-ICECS01_005.html

Valipour M, Ziatabar Ahmadi M, Raeini-Sarjaz M, Gholami Sefidkouhi MA, Shahnazari A, Fazlola R, Darzi-Naftchali A (2014) Agricultural water management in the world during past half century. Arch Agron Soil Sci. doi:10.1080/03650340.2014.944903

Xiaoying L, Erda L (2005) Performance of the Priestley-Taylor equation in the semiarid climate of North China. Agric Water Manag 71(1):1-17

Xu CY, Singh VP (2000) Evaluation and generalization of radiation-based methods for calculating evaporation. Hydrol Process 14(2):339-349

Ye J, Guo A, Sun G (2009) Statistical analysis of potential evapotranspiration on the Tibetan Plateau. J Irrig Drain Eng 135(2):134-140 